\title{
Adaptive Coupling of Resonators for Efficient Microwave Heating of Microfluidic Systems
}

\author{
Ali Amin Abduljabar, Student Member, IEEE, Heungjae Choi, Member, IEEE, David A. Barrow, and Adrian Porch
}

\begin{abstract}
An electronically adaptive coupling technique is presented for a microwave microstrip resonator to improve the efficiency of liquid heating in a microfluidic system. The concept is based on feeding the resonator with two synchronized inputs that have a variable phase shift between them. A Wilkinson power divider and phase shifter were designed and fabricated for this purpose. Both simulation and measurement (using chloroform as an exemplar liquid) demonstrated that the adaptive coupling can be used to optimize the heating efficiency of the liquid.
\end{abstract}

Index Terms-Adaptive coupling, microfluidic heating, microwave heating, split-ring resonator.

\section{INTRODUCTION}

$\mathbf{M}$ ICROWAVE heating techniques have been adopted and developed for many industrial, domestic, and medical applications. The many advantages of microwave heating can be summarized as: 1) high selectivity to absorbing components; 2) high efficiency of conversion of electromagnetic energy to heat when using microwave absorbing materials; 3) noncontacting energy delivery; and 4) volumetric heating. These advantages can be combined to offer potentially faster heating rates than for any other method [1]. Precision microfluidic heating control is required in many applications, such as for polymerase chain reactions (PCRs) [2], analysis of complex biological sample solutions [3], denaturizing dynamics of fluorescent proteins at the millisecond time scale [4], and the spatially localized heating of microchannel environments [5].

Much research has been undertaken to improve the heating performance when using microwave resonators as the applicator device in both large- and small-scale fluidic heating systems. In liter-sized liquid heating, for instance, improved electromagnetic heating of a load was demonstrated by increasing the isolation (or decoupling) factor between two electromagnetic feed elements [6]. In another study, very small magnetic particles were used to improve the microwave heating of biological samples [7].

Manuscript received April 20, 2015; revised July 16, 2015; accepted September 05, 2015.

A. A. Abduljabar was with the College of Engineering, University of Basrah, Basrah, Iraq. He is now with the School of Engineering, Cardiff University, Cardiff, CF 24 3AA, U.K. (e-mail: abduljabaraa@cardiff.ac.uk).

H. Choi, D. A. Barrow, and A. Porch are with the School of Engineering, Cardiff University, Cardiff, CF 24 3AA, U.K. (e-mail: choih1@cardiff.ac.uk; barrow@cardiff.ac.uk; porcha@cardiff.ac.uk).

Color versions of one or more of the figures in this paper are available online at http://ieeexplore.iee.org.

Digital Object Identifier 10.1109/TMTT.2015.2478004
A growing number of studies have focused on microfluidic heating systems, which is the subject of the work reported here. The performance of a planar microwave transmission line, integrated with a microfluidic channel to heat fluids with relevant buffer salt concentrations, was characterized and modeled over a wide range of frequencies [8]. An integrated microwave microfluidic heater that locally (and rapidly) increased the temperature of water drops in oil was demonstrated in [9]. A microwave heating system presented in [10] had a response time that was orders of magnitude faster than that of current commercial systems. In [11], an on-chip microwave generation of spatial temperature gradients was described within a polymeric microfluidic device that was coupled to an integrated microstrip transmission line. Moreover, a 2.45-GHz microwave cavity resonator was presented in [1] with the novel dual function of both sensitive dielectric characterization and directed volumetric heating of fluids in a microfluidic chip. Finally, a cost-effective scalable microwave system was demonstrated in [12] that can be integrated with microfluidic devices, thus enabling remote simultaneous sensing and heating of individual nanoliter-sized droplets generated in microchannels.

Normally, in a heating application the microwave input circuit is critically coupled to the resonant applicator device, resulting in maximum power transfer from source to applicator. The input impedance of the applicator can be written $Z_{\text {in }}=g$ - $Z_{0}$, where $g$ is the (dimensionless) coupling coefficient and $Z_{0}$ is the system impedance (usually $50 \Omega$, as is the case here). In practice $g$ depends on the geometrical details of the coupling structure, the unloaded $Q$ factor of the resonator, and the resonant frequency $f_{0}$. Critical coupling corresponds to the condition $g=1$, which is achieved for arbitrary values of $Q$ and $f_{0}$ by mechanical adjustment of the coupling structure. There are major challenges in heating materials in this way imposed by the temperature dependence of the properties of the materials themselves, which causes the $Q$ and (more seriously for a high- $Q$ resonator) $f_{0}$ to drift. For example, for the heating of aqueous samples, this is a result of the strong temperature dependence of the complex microwave permittivity of water. In summary, to maintain maximum efficiency during the heating process, we should be able to control: 1) excitation frequency and 2) coupling strength of the heating system simultaneously for continuous critical coupling condition. The excitation frequency can be easily controlled by signal source control while monitoring the response of the resonator. However, controlling coupling strength on a printed circuit board is not an easy task.

In this paper, a novel adaptive coupling topology is proposed that offers the ability to electronically adjust the coupling between the source and the heating resonator. The system con- 


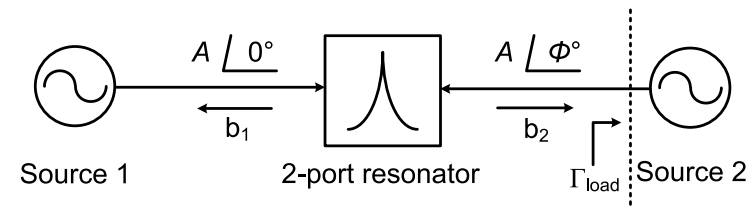

Fig. 1. Block diagram of a two-port resonator fed by two synchronized sources of the same frequency (equal to the resonant frequency) and with a phase shift of $\phi . A$ is the magnitude of the incident wave to the both ports of the resonator and $b_{1}$ and $b_{2}$ are the reflected waves from the resonator.

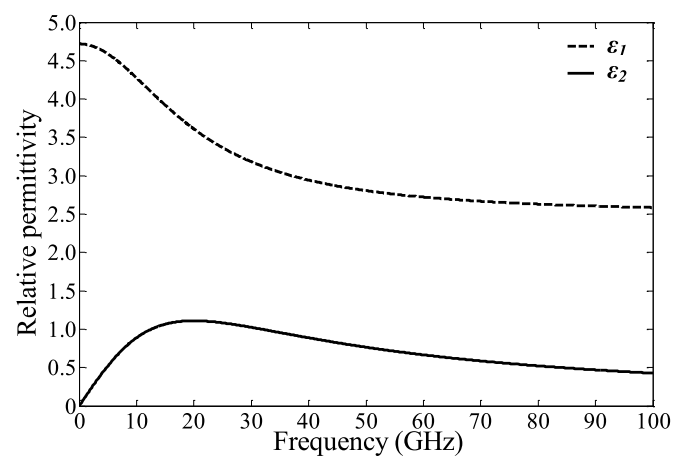

Fig. 2. Complex relative permittivity of chloroform at $25^{\circ} \mathrm{C}$, calculated from its Debye parameters listed in Table I.

sists of the resonator and two synchronized power sources with a variable phase shift between them, as shown in Fig. 1. A double split-ring resonator (DSRR) [13] was adopted as the host resonator and is connected to the two outputs of a variable phase-shift source. The adaptive coupling method was verified by heating chloroform as a test liquid within a quartz microcapillary.

The remainder of this paper is organized as follows. In Section II, the theories and concepts of microwave liquid heating, adaptive coupling resonator, design of two variable-phase sources, use of COMSOL Multiphysics, and temperature and complex permittivity measurements are described. In Section III, the design and realization of the microstrip resonator with two sources are described and analyzed. In Section IV, the simulated and experimental results are presented and discussed. In Section V, conclusions are drawn.

\section{THEORY AND CONCEPTS}

\section{A. Microwave Heating of Polar Liquids in Capillaries}

When placed in an oscillating electric field, the molecules of a polar liquid rotate owing to the torque generated by the field. This rotation, which continually changes its sense, results in dielectric heating due to friction. For common solvents, the heating is often most intense in the microwave frequency range. The relative permittivity of the liquid can be written as a complex number $\varepsilon=\varepsilon_{1}-j \varepsilon_{2}$ with real and imaginary parts well described by the Debye theory. The complex permittivity of our test liquid chloroform is plotted in Fig. 2 based on its Debye parameters listed in Table I. From Fig. 2, the maximum loss occurs at $20 \mathrm{GHz}$, where $\varepsilon_{2}=1.11$. The dissipated power density within the liquid is given by [14]

$$
p=2 \pi f \varepsilon_{o} \varepsilon_{2}|E|^{2}
$$

TABLE I

Material Properties Used In the Simulation at $25{ }^{\circ} \mathrm{C}$

\begin{tabular}{cccc}
\hline \hline Material & $\varepsilon_{1}$ & $\varepsilon_{2}$ & $\sigma(\mathrm{S} / \mathrm{m})$ \\
\hline Air & 1 & 0 & 0 \\
Microstrip Dielectric & 2.2115 & 0 & $3.17 \times 10^{-4}$ \\
Copper & 1 & 0 & $2.7 \times 10^{7}$ \\
Quartz Capillary & 3.4 & 0 & $1 \times 10^{-12}$ \\
\hline
\end{tabular}

Chloroform [18]

\begin{tabular}{ccccccc}
$\varepsilon_{s}$ & $\varepsilon_{\infty}$ & $\begin{array}{c}\tau \\
(p \mathrm{~s})\end{array}$ & $\begin{array}{c}\mathrm{k} \\
(\mathrm{W} /(\mathrm{m} \cdot \mathrm{K}))\end{array}$ & $\begin{array}{c}\rho \\
\left(\mathrm{kg} / \mathrm{m}^{3)}\right.\end{array}$ & $\begin{array}{c}\mathrm{C}_{\mathrm{p}} \\
(\mathrm{J} /(\mathrm{kg} \cdot \mathrm{K}))\end{array}$ & $\gamma$ \\
\hline 4.72 & 2.5 & 7.96 & 0.1152 & 1489 & 1050 & 1.11 \\
\hline \hline
\end{tabular}

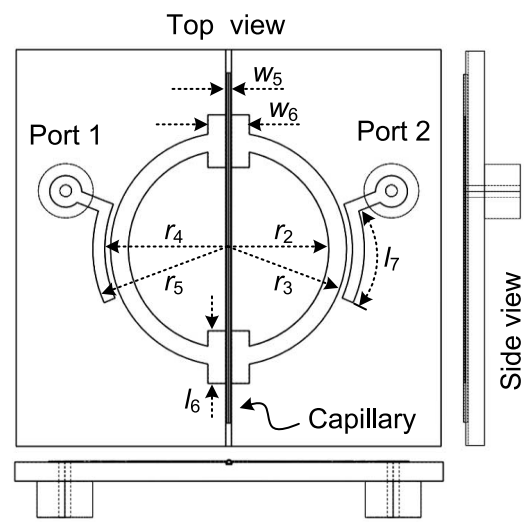

Fig. 3. Definition of the symbols used to define the dimensions of the DSRR structure, also including the coupling ports.

where $E$ is the electric field amplitude within the liquid and $f$ is the excitation frequency. The DSRR used in this heating study is shown diagrammatically in Fig. 3, similar to that described in [13] for microwave sensing, but with modifications for this heating application. The two portions of liquid within the two gap regions will be heated.

In our DSRR, the electric field is perpendicular to the axis of the liquid sample (assumed cylindrical since it is held within a cylindrical capillary), which means that $E$ is reduced greatly compared to the amplitude of the applied field $E_{o}$ owing to the effects of depolarization.

This is dealt with in detail in [15], but to a good approximation $E$ is found to be

$$
E=\frac{4 \varepsilon_{r T} E_{o}}{\left(\varepsilon_{r T}+1\right)\left(\varepsilon_{r L}+\varepsilon_{r T}\right)+\left(\varepsilon_{r T}-1\right)\left(\varepsilon_{r L}-\varepsilon_{r T}\right) a^{2} / b^{2}}
$$

where $\varepsilon_{r T}$ and $\varepsilon_{r L}$ are the relative permittivity of the capillary (tube) and the liquid, respectively, while $a$ and $b$ are the inner and outer radii of the capillary, respectively, as shown in Fig. 4. It is clear from (1) and (2) that the heat efficiency is inversely proportional to the real part of the liquid and directly proportional to the imaginary part of the liquid's relative permittivity.

\section{B. Adaptive Coupling Method}

The aim of our adaptive coupling method is to decrease or increase the coupling between the resonator and the input ports 


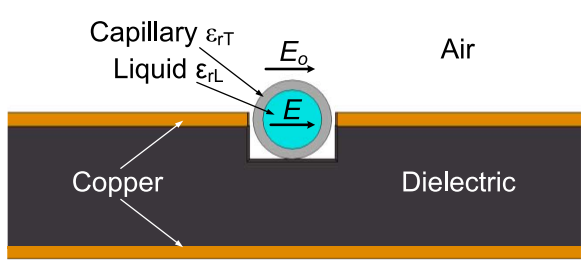

Fig. 4. Cross section of the capillary inside the resonator gap, showing schematically the applied electric field $E_{0}$ and the resulting electric field $E$ within the capillary.

TABLE II

Dimensions of the ADAPTIVE COUPLING DSRR SHOWn IN Figs. 3 AND 14. All Dimensions ARE IN Millimeters

\begin{tabular}{cccccccc}
\hline \hline$i$ & 1 & 2 & 3 & 4 & 5 & 6 & 7 \\
\hline$\ell_{i}$ & 28.00 & 16.20 & 20.75 & 18.45 & 46.85 & 4.50 & 8.04 \\
$\mathrm{w}_{i}$ & 4.77 & 2.78 & 7.33 & 4.96 & 0.46 & 3.50 & 5.02 \\
$\mathrm{r}_{i}$ & 10.84 & 8.50 & 10.00 & 10.50 & 11.50 & ----- & ----- \\
\hline \hline
\end{tabular}

electronically. This provides the ability to control the power delivery to the resonator, and consequently, the ability to the control heating without changing the source power.

On varying the phase shift between the two, equal-power inputs at ports 1 and 2 (as in the schematic of Fig. 1), the total delivered power to the resonator changes. To verify this concept of adaptive resonator coupling, a simulation of the DSRR shown schematically in Fig. 3 (with dimensions shown in Table II) was performed using COMSOL Multiphysics for the microwave heating of chloroform. If an input power of $P_{i}$ is applied to one of the input ports of the resonator, the dissipated power in the liquid is calculated to be $0.24 P_{i}$ at the resonant frequency $(2.861 \mathrm{GHz})$, while the rest of the power $0.76 P_{i}$ is dissipated in the rest of the DSRR structure (mostly the copper parts) and also lost by radiation.

If the power $P_{i}$ is halved to two parts $\left(P_{i} / 2+P_{i} / 2\right)$ and fed into the two inputs simultaneously with variable phase shift between them, then the delivered dissipated power in the liquid can be controlled by varying the phase. It is clear from Fig. 5 that if the phase shift between two inputs is 0 , the dissipated power in chloroform will be zero, and can be increased to up to $0.48 P_{i}$ when the phase shift is $180^{\circ}$ at the resonant frequency $(2.861 \mathrm{GHz})$. This is double the value of dissipated power compared with the case when the same value of power is applied to one port only. For $90^{\circ}$ phase difference, the same power is dissipated in the liquid as when the power is applied to one port only. To explain why maximum power dissipation happens when the two sources are $180^{\circ}$ out of phase, the source that feeds port two with variable phase is used as variable load impedance. If we assume the two port S-parameters of the resonator as $\mathrm{S}_{11}, \mathrm{~S}_{12}, \mathrm{~S}_{21}$, and $\mathrm{S}_{22}$, as shown in Fig. 1, the reflection coefficient of the load $\Gamma_{\text {load }}$ can be given as

$$
\Gamma_{\text {load }}=\frac{A e^{j \phi}}{b_{2}}=\frac{1}{S_{21} \frac{A}{A e^{j \phi}}+S_{22}}=\frac{1}{S_{21} e^{-j \phi}+S_{22}} .
$$

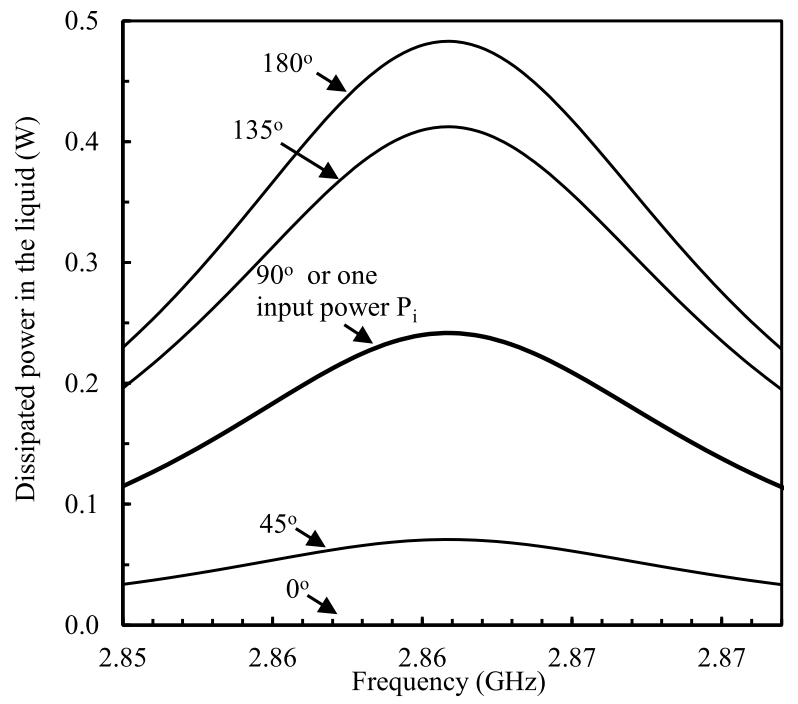

Fig. 5. Simulated power dissipation in the liquid (chloroform) as a function of frequency with respect to the phase shift between the two microwave inputs, calculated using COMSOL Multiphysics.

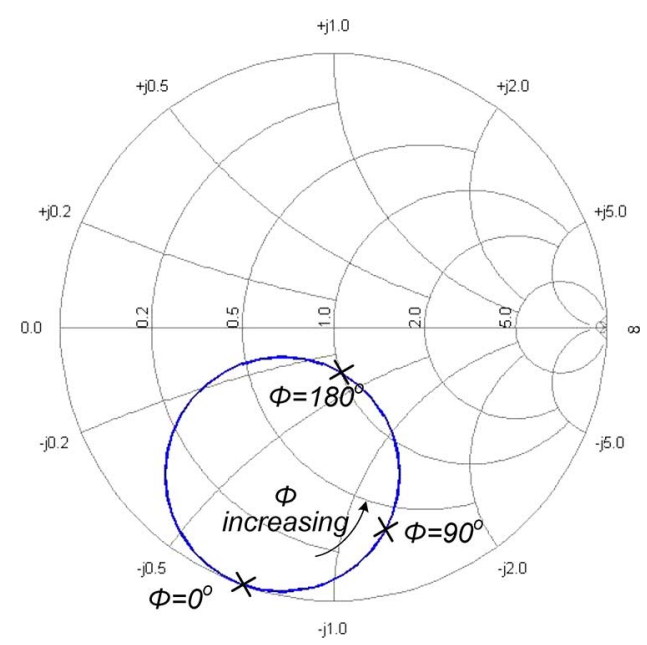

Fig. 6. Simulated load impedance (blue line) of the DSRR with variable phase output source.

It can be seen from (3) that the value of $\Gamma_{\text {load }}$ can be controlled by varying the value of the phase shift $\phi$. Therefore the source of port 2 is utilized to change the load impedance of the resonator to the optimum value in order to increase the matching between the sources and the two inputs of the resonator.

The load impedance, which was obtained from the simulation with the different phase shift between two sources (from $0^{\circ}$ to $360^{\circ}$ ) at the resonant frequency $2.861 \mathrm{GHz}$ were plotted in the Smith chart, as shown in Fig. 6. From Fig. 6, it can be seen that the optimum matching occurs at $\phi=180^{\circ}$ where the maximum power is delivered to the resonator.

The experimental realization of a variable phase-shift twoinput resonator can be achieved by using a Wilkinson power divider (to split the power into two equal parts) and a quadrature phase-shifter connected to varactors [16]. The varactor capacitance is varied with an applied dc bias voltage, which changes the phase by imbalancing the otherwise equal electric lengths of 


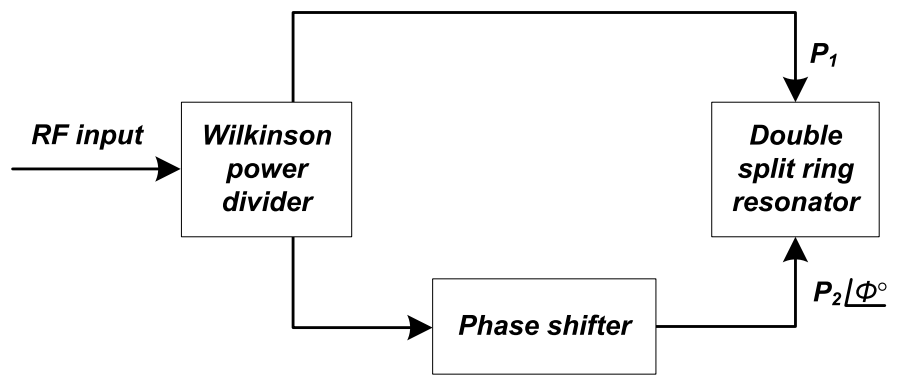

Fig. 7. Block diagram of the DSRR fed with input signals with different phases achieved by using an adjustable phase-shifter circuit.

the microstrip sections [17]. The block diagram illustrating this type of adaptive coupling is shown in Fig. 7.

\section{Use of COMSOL Multiphysics}

COMSOL Multiphysics 4.4 was used to perform 3-D simulations of the electromagnetics, electromagnetics with lumped elements, and coupled electromagnetic-thermal effects. The electromagnetic waves model was used to simulate the S-parameters, dissipated power in the liquid (chloroform), and the input/ output impedances of the resonator. The wave equation in the frequency domain was computed in the electromagnetic waves model described in the software as

$$
\nabla \times \mu_{r}^{-1}(\nabla \times \bar{E})-k_{o}^{2}\left(\varepsilon_{r}-\frac{j \sigma}{\omega \varepsilon_{o}}\right) \bar{E}=0
$$

$\mu_{r}$ is the permeability, $\varepsilon_{r}$ is the permittivity, and $\sigma$ is the electric conductivity of the material; $\varepsilon_{o}$ is the permittivity of the vacuum, $k_{o}$ is the wavenumber in free space, and $\omega$ is the wave angular frequency. The impedance boundary condition is used for the copper surfaces of the resonator and ground in order to consider the copper losses. The scattering boundary condition was utilized for the faces of the volume $80 \times 125 \times 80 \mathrm{~mm}^{3}$ (enclosing the device) to make the boundaries transparent for the scattered waves. Coaxial ports were used to feed the electromagnetic energy to the resonator and lumped elements were used to set the variable reactor of the varactors.

The microwave heating model was used to obtain the heat distribution in the liquid as defined in the software

$$
\rho C_{p} \bar{u} \cdot \nabla T=\nabla \cdot(k \nabla T)+Q
$$

where $\rho$ is the density of the material, $C p$ is the heat capacity at constant pressure, and $k$ is the thermal conductivity of the material. $\bar{u}$ is the spatial displacement vector, $T$ is the temperature, and $Q$ is the heat source. For Joule heating, this is driven by the electric current and is added in the electromagnetic heat source node.

The relative permittivity of chloroform was described in the simulation by using Debye theory as its permittivity is variable with frequency. The properties of the materials that are used in the simulation are shown in Table I.

In Table I, $\varepsilon_{s}$ and $\varepsilon_{\infty}$ are the values of permittivity in the static $(\omega \tau \ll 1)$ and $\operatorname{VHF}(\omega \tau \gg 1)$ limits, respectively, $\tau$ is the relaxation time, and $\gamma$ is the ratio of specific heats. A maximum

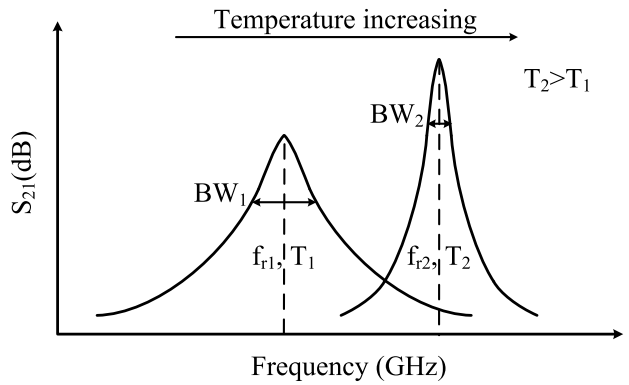

Fig. 8. Variation of $S_{21}$ with temperature due the change in complex permittivity.

mesh size for the simulations was set to be $3 \mathrm{~mm}$, while the minimum mesh size is $0.24 \mathrm{~mm}$, which produced $211166 \mathrm{mesh}$ elements.

\section{Temperature and Complex Permittivity Measurements}

There are several methods to measure the temperature of liquid in the microfluidic systems, such as the microscopy fluorescence thermometry technique [19]. Our approach is based on the fact that the complex permittivity varies with temperature as well as frequency. Complex permittivity can be defined as in [20]-[22]

$$
\varepsilon(T, \omega)=\varepsilon_{1}(T, \omega)-j \varepsilon_{2}(T, \omega)
$$

where

$$
\varepsilon_{1}(T, \omega)=\varepsilon_{\infty}(T)+\frac{\varepsilon_{s}(T)-\varepsilon_{\infty}(T)}{1-\omega^{2} \tau^{2}(T)}
$$

and

$$
\varepsilon_{2}(T, \omega)=\frac{\left(\varepsilon_{s}(T)-\varepsilon_{\infty}(T)\right) \omega \tau(T)}{1-\omega^{2} \tau^{2}(T)}
$$

then the value of the complex permittivity can be linked with the temperature. In polar liquids such as chloroform, the $\varepsilon_{1}$ and $\varepsilon_{2}$ values both decrease with temperature. In our design, the resonant frequency and quality factor depend on the liquid sample, modeled by first-order cavity perturbation concepts [23]. Therefore, the change in temperature causes a shift in resonant frequency and change in quality factor (or, equivalently, 3-dB bandwidth), as shown in Fig. 8, due to the change in liquid complex permittivity, which can then be used to obtain the liquid's average temperature.

We measured the complex permittivity of chloroform with temperature at a frequency of $2.861 \mathrm{GHz}$, as described in Section IV. When the liquid is heated by the resonator, the resonant frequency increases from $f_{r 1}$ to $f_{r 2}$, and in addition the bandwidth decreases from $\mathrm{BW}_{1}$ to $\mathrm{BW}_{2}$, as illustrated in Fig. 8. From these shifts in resonant frequency and bandwidth, the corresponding change in the complex permittivity can be determined. A routine involving simulation has been applied to find the exact value of the complex permittivity for the new value of the resonant frequency and bandwidth $\left(f_{r 2}, \mathrm{BW}_{2}\right)$. A new value of complex permittivity found from the simulation is compared with the measured values of complex permittivity in order to deduce the temperature. 


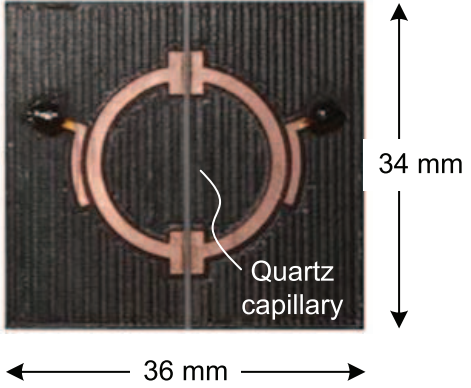

Fig. 9. Photograph of the microstrip heating resonator (DSRR), fabricated by milling a Rogers RT/Duroid 5880 laminate.

\section{DESIGN AND REALIZATION}

The DSRR described in [13] was adopted and modified for this study. The input/output coupling was strengthened by reducing the gap between it and the DSRR, to increase the delivered power to the liquid.

The design of the resonator was aided by COMSOL Multiphysics simulations. A photograph of the fabricated DSRR is shown in Fig. 9, designed to heat chloroform within a quartz capillary at $2.861 \mathrm{GHz}$. The design procedures of the Wilkinson power divider and the quadrature phase shifter were taken from [23], also to operate at the center frequency of $2.861 \mathrm{GHz}$ (the resonant frequency of the DSRR). The circuits were fabricated by the mechanical milling of a Rogers Corporation RT/Duroid 5880 laminate, with a dielectric of a thickness of $1.57 \mathrm{~mm}$, relative permittivity of $2.20 \pm 0.02$, and loss tangent of 0.0009 . The thickness of the copper was chosen as $70 \mu \mathrm{m}$ to ensure the highest possible quality factor for the DSRR. The dimensions of Wilkinson power divider and quadrature phase shifter were calculated by a line calculation tool in Advanced Design System 2014 (Keysight Technology). However, due to discontinuities, junction effects, or unequal even- and odd-mode velocities, the performances of the Wilkinson power divider and quadrature phase shifter became degraded in the simulation [24], [25]. Slight modifications of their lengths $\ell$, and widths $w$, were made to improve their simulated performances. Table II shows the dimensions of all designed components.

The simulated S-parameters of the Wilkinson power divider illustrated in Fig. 10 are shown in Fig. 11, where good input impedance-matching characteristics are observed and the coupled power at each output port is about $-3.2 \mathrm{~dB}$ around $2.861 \mathrm{GHz}$, with a phase delay of $90^{\circ}$. The dimensions of the Wilkinson power divider are illustrated in Table II.

The form of the quadrature $\left(90^{\circ}\right)$ hybrid coupler is shown schematically in Fig. 12, which is tuned to operate at $2.861 \mathrm{GHz}$. The simulated S-parameters of the quadrature hybrid coupler are shown in Fig. 13 (using COMSOL Multiphysics). The S-parameters of the branch line coupler show good input matching $\mathrm{S}_{11}$ and isolation $\mathrm{S}_{41}$ around $2.861 \mathrm{GHz}$, where the coupled signal at the two output ports $\left(\mathrm{S}_{21}\right.$ and $\left.\mathrm{S}_{31}\right)$ is at about $-3.2 \mathrm{~dB}$ at $2.861 \mathrm{GHz}$. Fig. 13(b) shows the phase shift (in degrees) between the outputs (ports 2-4) and the input port 1. The phase shift between ports 2 and 3 is about $90^{\circ}$ at $2.861 \mathrm{GHz}$, where in the ideal phase shift is equal to $90^{\circ}$.

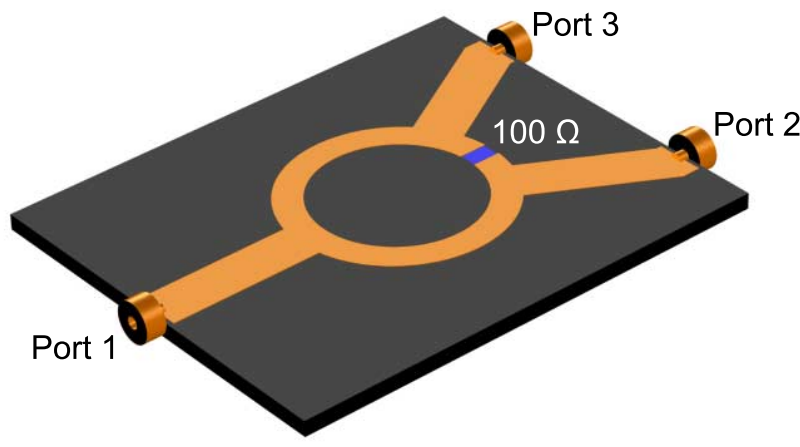

Fig. 10. Model of the Wilkinson power divider, which was simulated using COMSOL Multiphysics.

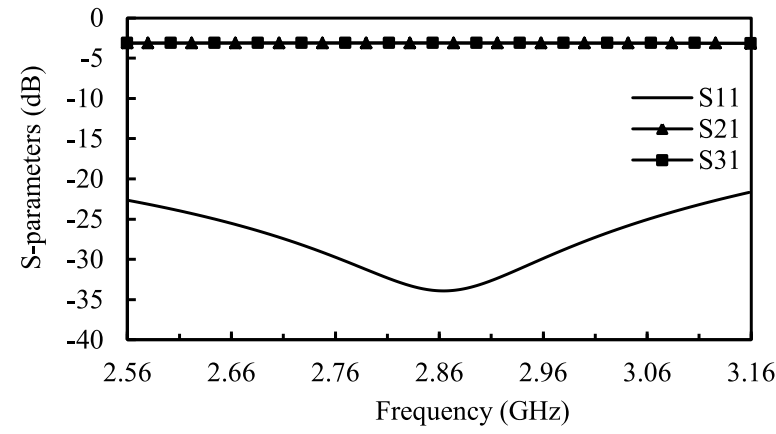

a

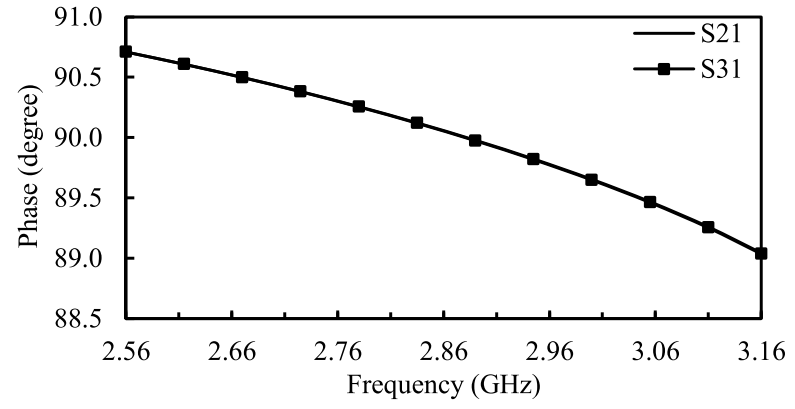

$\mathrm{b}$

Fig. 11. (a) S-parameters of the Wilkinson power divider are simulated using COMSOL Multiphysics, show good input matching at $2.861 \mathrm{GHz}$, and evenly divided power at the two output ports. (b) Phases of the two output ports show approximately $90^{\circ}$ difference at $2.861 \mathrm{GHz}$.

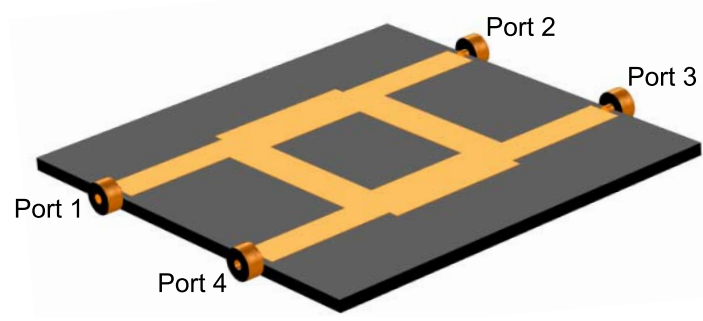

Fig. 12. Model of a quadrature $\left(90^{\circ}\right)$ hybrid coupler is simulated by COMSOL Multiphysics.

A phase shifter can be achieved by incorporating the quadrature hybrid coupler with variable capacitors or inductors to obtain a variable phase shifter. Variable capacitors or inductors 


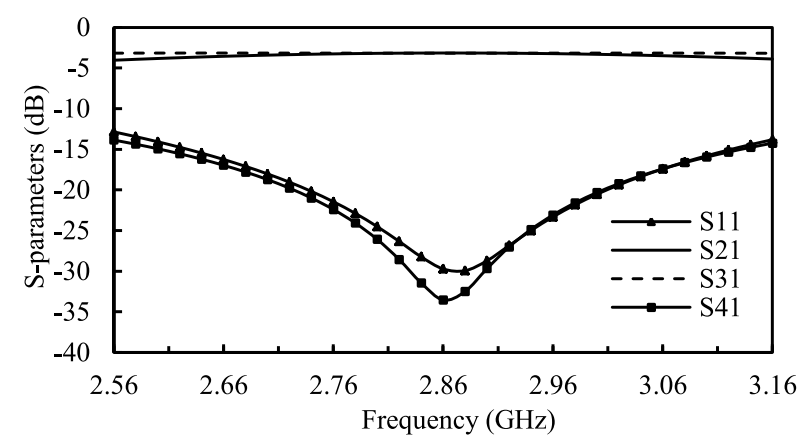

a

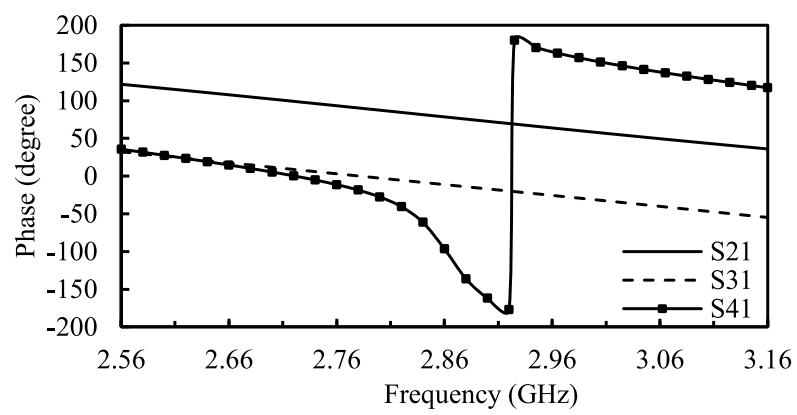

b

Fig. 13. (a) S-parameters of a quadrature $\left(90^{\circ}\right)$ hybrid coupler are simulated by COMSOL Multiphysics. (b) Similar plot for the phases.

were connected to ports 2 and 3 of the quadrature hybrid coupler, which act as short circuits to reflect the incident energy back towards port 4 with phase shift depending on the their values. A variable capacitor can be realized by a varactor diode in which the variable phase can be controlled electronically, as shown in the full circuit topology illustrated in Fig. 14, which also shows a photograph of the final circuit.

As the active volume of liquid in our system is tiny, only $0.64 \mu \mathrm{L}$ in both gaps, the dissipated power required to heat chloroform is less than $1 \mathrm{~W}$, which produces a heating rate around $60{ }^{\circ} \mathrm{C} / \mathrm{s}$. A varactor diode 1T362A (Sony Corporation) was chosen, which can handle this small amount of power. In reverse bias, a varactor diode yields a capacitance range of approximately $2.3-100 \mathrm{pF}$ over $25-0 \mathrm{~V}$ at $900 \mathrm{MHz}$, respectively [26]. However, at $2.861 \mathrm{GHz}$, the variable reactor of the diode has an inductive effect as the frequency of operation is higher than the self-resonant frequency, which makes the package inductance of the diode dominant. The measured reactance of the diode in reverse bias at $2.861 \mathrm{GHz}$ is shown in Fig. 15. In the design it was required to set the phase-shift range with the range of the variable inductor. An $180^{\circ}$ phase shift between ports 2 and 3 was achieved by setting the diode's effective inductance to a value of $0.7 \mathrm{nH}$.

This setting is realized by carefully choosing $\ell_{1}$ to be $28 \mathrm{~mm}$, which gives a phase shift of $132^{\circ}$ at $2.861 \mathrm{GHz}$. Together with the quadrature $\left(90^{\circ}\right)$ hybrid coupler and the two parallel diode inductors, the phase shift between ports 2 and 3 at $2.861 \mathrm{GHz}$ will then be $180^{\circ}$.

The groove for the quartz capillary was fabricated using laser micromachining with a width of $\mathrm{w}_{5}$ (i.e., $0.46 \mathrm{~mm}$ ) and depth of $0.235 \mathrm{~mm}$. Microwave connection to the input port (port 1 at the top of Fig. 14) was made via the dielectric layer and the
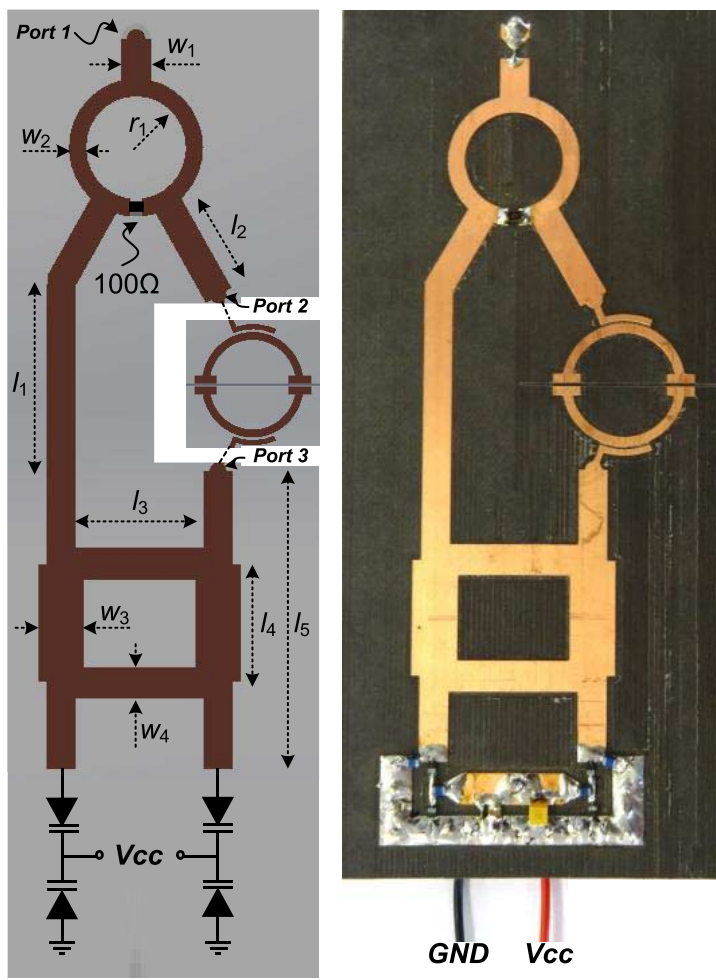

Fig. 14. Circuit topology and photograph of the DSRR connected to the two outputs of a variable phase-shift power source that consisted of the Wilkinson power divider, quadrature phase shifter, and varactor circuit.

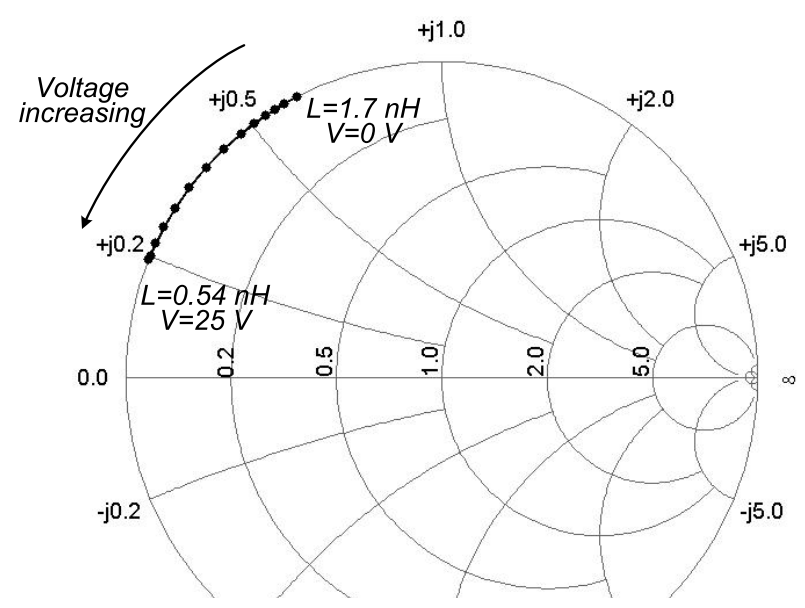

Fig. 15. Measured variable inductance of the varactor 1T362A at $2.861 \mathrm{GHz}$.

ground of the microstrip board using a surface-mounted coaxial (SMA) connector. The quartz capillary has an inner diameter of $0.3 \mathrm{~mm}$ and outer diameter of $0.4 \mathrm{~mm}$.

The capillary was inserted in the groove and filled by liquid using capillary action [13]. Chloroform was selected as the test liquid, as it is a high-loss polar liquid with a low boiling point $\left(61.5{ }^{\circ} \mathrm{C}\right)$, which does not demand a high-power microwave source to heat it. Separate resonant cavity measurements of chloroform were used to determine its complex permittivity of $\varepsilon=4.80-j 0.30$ (with an error of about $2 \%$ in both real and imaginary parts) at $25^{\circ} \mathrm{C}$ at $2.861 \mathrm{GHz}$. This value was used in the COMSOL simulations for the design of the DSRR. The resulting DSRR with a chloroform sample was measured 


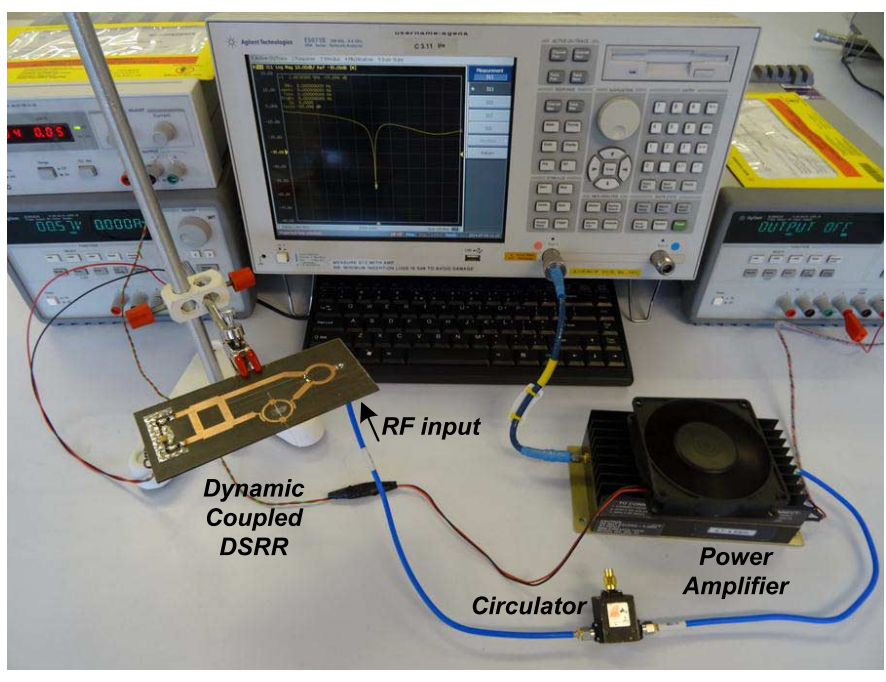

Fig. 16. Photograph the bench-top experimental assembly. The microwave source was provided by an Agilent E5071B network analyzer. Other components are labeled.

to have a resonant frequency of $2.861 \mathrm{GHz}$ and an unloaded quality factor of 150 .

To deliver the power to the adaptive coupling resonator circuit, a power amplifier ZHL-42 (manufactured by Mini-Circuits) was used to amplify the signal from an Agilent E5071B network analyzer. The power amplifier gain is $29.6 \mathrm{~dB}$ and the range of delivered power from the network analyzer is from -10 to $0 \mathrm{dBm}$. The resulting input power to the circuit is from 20 to $30 \mathrm{dBm}$, which covers the power range required to boil the chloroform sample. A circulator was used to protect the power amplifier from the reflected power. Fig. 16 shows the bench-top assembly of the adaptive coupling resonator with the power amplifier and the network analyzer.

\section{Results AND Discussion}

To verify the ability of the resonator with adaptive coupling to increase the heating efficiency, as well as controlling strength of the coupling, tests were undertaken, firstly by feeding only one input port of the resonator, which incorporated a chloroform filled capillary. This was heated to boiling at different values of input power.

Due to the difficulty of measuring the liquid temperature inside the resonator-integrated capillary, the shift in the resonant frequency was used to measure the temperature of chloroform, as the permittivity of a polar liquid is a strong function of temperature [27]. The complex permittivity of heated chloroform can be calculated by applying an optimization routine based on matching the simulated and experimental results (see Section II-D). The temperature can then be determined from the new value of liquid permittivity.

As previously mentioned, a separate microwave cavity was used to measure the complex permittivity of chloroform using standard cavity perturbation analysis [23] and [28]. This can be done over a variable temperature range by placing the cavity in an oven (Memmert, Model: IPP 400) with a high degree of temperature control $\left( \pm 0.1^{\circ} \mathrm{C}\right)$.

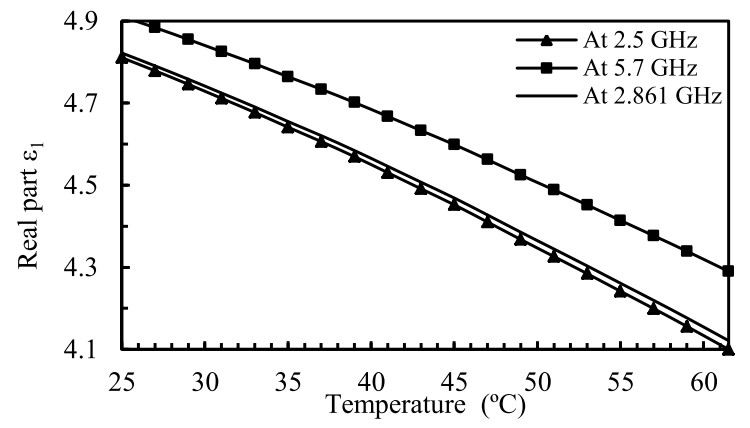

a

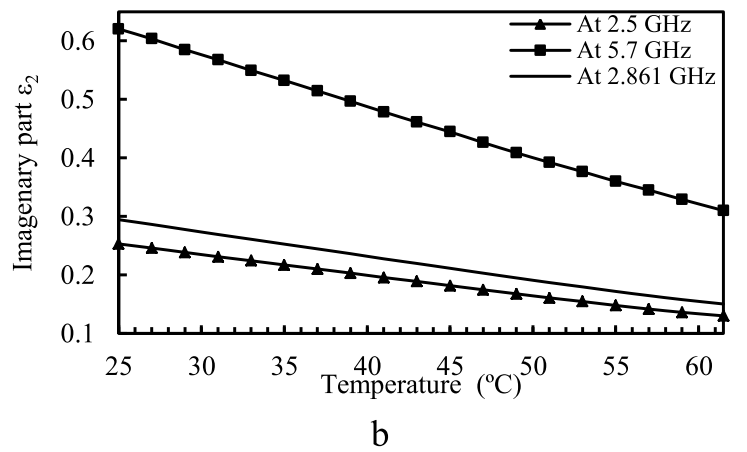

Fig. 17. Cavity measurements of the complex permittivity of chloroform with temperature. (a) Real part and (b) imaginary part at frequencies of 2.5 and $5.7 \mathrm{GHz}$. The values shown at $2.861 \mathrm{GHz}$ are linearly interpolated between these two measurement frequencies.

The complex permittivity of chloroform was measured over the temperature range of $25.0{ }^{\circ} \mathrm{C}-61.5{ }^{\circ} \mathrm{C}$ (i.e., to boiling) at the two resonant frequencies of the $\mathrm{TM}_{010}$ and $\mathrm{TM}_{020}$ modes of the cylindrical cavity (at 2.5 and $5.7 \mathrm{GHz}$, respectively). Results at $2.861 \mathrm{GHz}$ were found by linearly interpolating the complex permittivity between 2.5 and $5.7 \mathrm{GHz}$. These are plotted as a function of temperature in Fig. 17. By knowing the complex permittivity of chloroform as a function of temperature, the temperature of the chloroform can be inferred with a high degree of accuracy from measuring the resonant frequency of the DSRR (or indeed any type of resonant applicator used to heat it).

As shown in Fig. 18, the resonant frequency is $2.8610 \mathrm{GHz}$ when $1 \mathrm{~mW}$ of power was applied to only one input port of the DSRR. This means that there was negligible heating of the chloroform at such low powers and the chloroform temperature remained at $25{ }^{\circ} \mathrm{C}$ (room temperature). By increasing the input power to $180 \mathrm{~mW}$, boiling occurred when the resonant frequency increased to $2.8631 \mathrm{GHz}$, which accurately predicts the boiling point of chloroform of $61.5^{\circ} \mathrm{C}$ (where the real permittivity is 4.10 ).

In the second part of the experiments, the DSRR was fed with two input signals of variable phase difference with the circuits shown in Figs. 14 and 16. The phase shift between ports 2 and 3 shown in Fig. 14 was measured with variable bias voltages applied to the varactor diode, as shown in Fig. 19. Here the DSRR was removed and the output was terminated with a $50-\Omega$ load. At the resonant frequency of the DSRR $(2.861 \mathrm{GHz})$ with the chloroform at $25^{\circ} \mathrm{C}$, the circuit provides a range of phase shifts from $128^{\circ}$ at $0 \mathrm{~V}$ to $190^{\circ}$ at $25 \mathrm{~V}$. 


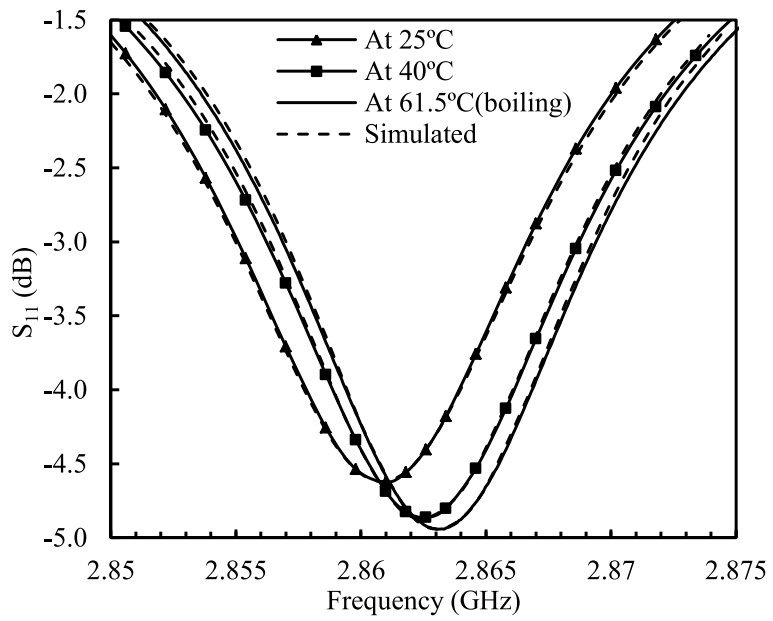

Fig. 18. Simulated and measured magnitudes of the voltage reflection coefficients $\mathrm{S}_{11}$ for the single-port DSRR at different temperature degrees.

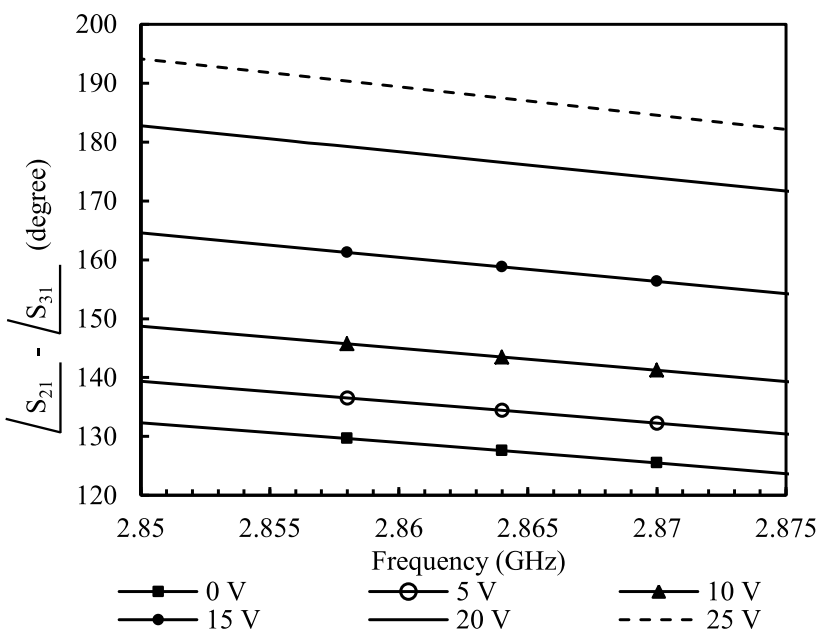

Fig. 19. Measured phase of $\mathrm{S}_{23}$ between Ports 2 and 3 of Fig. 14 for different values of the applied bias voltage.

This covers the condition for maximum power transfer into the DSRR that occurs at $180^{\circ}$ according to the simulation shown in Fig. 5.

To determine the performance of the adaptive coupling resonator shown in Fig. 14 for optimum heating efficiency, microwave power was fed to the input port of the circuit shown in Fig. 16. At each input power, the bias voltage was adjusted to give the maximum coupling, i.e., maximum power transfer, corresponding to a phase difference of $180^{\circ}$, as shown in Fig. 20. The input power required for boiling (where the DSRR's resonant frequency was shifted to $2.6831 \mathrm{GHz}$ due to the change in permittivity), was $45 \mathrm{~mW}$ at each port (port 2 or 3 ) of the DSRR, or $90 \mathrm{~mW}$ when fed at both ports. After heating the chloroform to $61.5^{\circ} \mathrm{C}$, the return loss decreased by $4 \mathrm{~dB}$ due to a combination of the increase in resonant frequency and increased value of quality factor.

The coupling can be readjusted to return to the same value of return loss $(49 \mathrm{~dB})$ attained before heating. Moreover, as shown in Fig. 20, the three dotted lines and maximum coupling line correspond to the magnitudes of $S_{11}$ with different values of

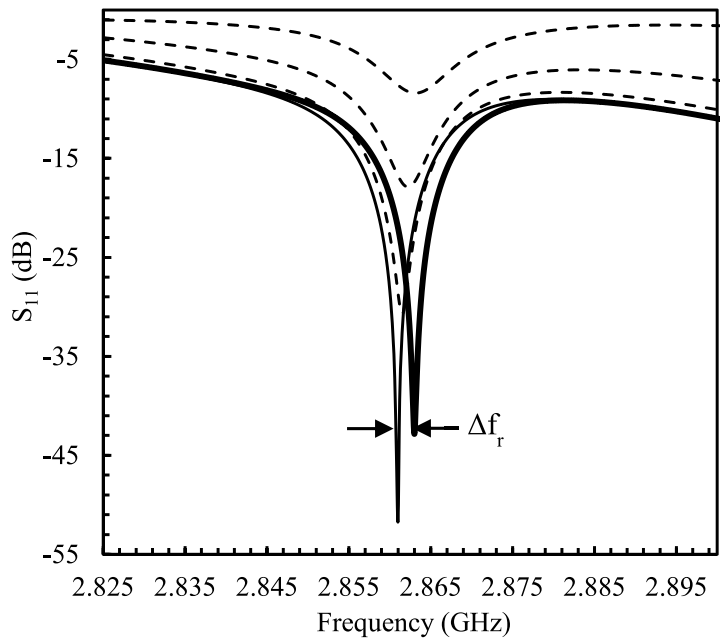

- At max. Coupling, $180 \mathrm{deg}$ and $0 \mathrm{dBm}$

At boiling temperature $\left(61.5^{\circ} \mathrm{C}\right), 180 \mathrm{deg}$ and $19.54 \mathrm{dBm}$ - - - At variable coupling and $0 \mathrm{dBm}$

Fig. 20. Measured values of $S_{11}$ of the adaptive coupling resonator in Fig. 16.

TABLE III

Performance Comparison Between the Two Types of Power Feeding

\begin{tabular}{ccccc}
\hline \hline & $\begin{array}{c}\text { Input power } \\
(\mathrm{mW})\end{array}$ & $\Delta \mathrm{f}(\mathrm{MHz})$ & $\begin{array}{c}\text { Complex } \\
\text { permittivity }\end{array}$ & $\begin{array}{c}\text { Temperature } \\
\left({ }^{\circ} \mathrm{C}\right)\end{array}$ \\
\hline $\begin{array}{c}\text { One port } \\
\text { feeding }\end{array}$ & 90 & $1.2 \pm 0.1$ & $4.55-\mathrm{j} 0.23$ & 40 \\
\hline $\begin{array}{c}\text { Two } \\
\text { ports } \\
\text { feeding }\end{array}$ & $\begin{array}{c}90 \\
\text { (both ports) }\end{array}$ & $2.0 \pm 0.1$ & $4.10-\mathrm{j} 0.15$ & 61.5 \\
\hline \hline
\end{tabular}

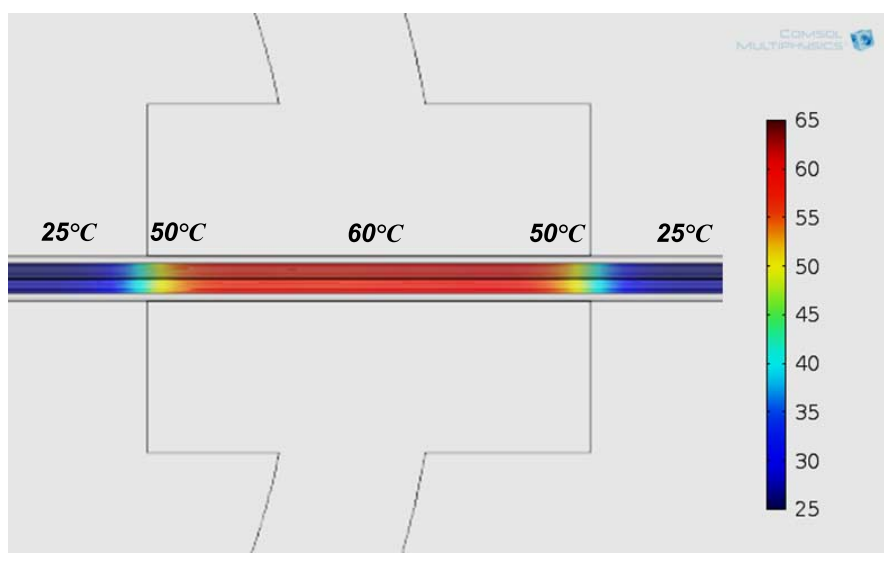

Fig. 21. Simulated temperature distribution over the channel for the one input DSRR with $180 \mathrm{~mW}$, or two input DSRR with $45 \mathrm{~mW}$ at each input.

diode dc voltage and $0-\mathrm{dBm}$ input power. This means that the coupling changes with the value of the dc-bias voltage, demonstrating the ability to control the coupling electronically.

A summary of the adaptive coupling circuit performance is illustrated in Table III. As shown in Fig. 20, there is a change in resonant frequency due to the coupling variation. The resonant frequency changes are approximately $2.0 \pm 0.1 \mathrm{MHz}$, which is well within the bandwidth of the power amplifier. Finally, a simulation of the heat distribution along the chloroform 
sample was undertaken using COMSOL Multiphysics to illustrate the distribution of the temperature along the gap area, as shown in Fig. 21. This simulation is when the DSRR is fed with $180 \mathrm{~mW}$ at one port, or $45 \mathrm{~mW}$ at two ports, with a phase shift of $180^{\circ}$ between them (total input power is $90 \mathrm{~mW}$ ). In both cases, the chloroform was heated to boiling along the center region $\left(61.5^{\circ} \mathrm{C}\right)$.

\section{CONCLUSION}

A novel adaptive coupling method that provides the ability to change (and, in principle, control) the coupling of a microwave resonator electronically has been presented in this paper. This approach can be exploited in microfluidic heating applications, where the heating rate can be optimized without changing the source power. The power gain, or the extent of heating, can be increased to double the power value of that used to feed a one-port resonator. For example, with chloroform, the total power (applied to both ports) required to reach the boiling temperature was half the value needed in the one-port configuration. The verification of this new concept was achieved by simulation and experiment, including the dissipated power, the heat distribution of the liquid, S-parameter measurements, and the associated shifts in resonant frequency. The proposed topology is used for proof-of-principle, and as a future work, miniaturization will be achieved by using commercially available power dividers and quadrature hybrid couplers.

\section{REFERENCES}

[1] A. J. L. Morgan et al., "Efficient microwave heating of microfluidic systems," Sens. Actuators B, vol. 181, pp. 904-909, May 2013.

[2] J. Liu, M. Enzelberger, and S. Quake, "A nanoliter rotary device for polymerase chain reaction," Electrophoresis, vol. 23, no. 10, pp. 1531-1536, May 2002.

[3] R.-H. Liu, J. Yang, R. Lenigk, J. Bonanno, and P. Grodzinski, "Self contained, fully integrated biochip for sample preparation, polymerase chain reaction amplification, and DNA microarray detection," Anal. Chem., vol. 76, no. 7, pp. 1824-1831, Feb. 2004.

[4] H.-F. Arata, F. Gillot, T. Nojima, T. Fujii, and H. Fujita, "Millisecond denaturation dynamics of fluorescent proteins revealed by femtoliter container on micro-thermidevice," Lab Chip, vol. 8, no. 9, pp. 1436-1440, Sep. 2008.

[5] A.-J. de Mello, M. Habgood, L. Lancaster, T. Welton, and R.-C. Wootton, "Precise temperature control in microfluidic devices using Joule heating of ionic liquids," Lab Chip, vol. 4, no. 5, pp. 417-419, Jul. 2004.

[6] I. Chaimov and S. R. Rogers, "Electromagnetic heating apparatus having decoupled excitations," in IEEE Int. Microw., Commun., Antennas, Electron. Syst. Conf., Tel Aviv, Israel, 2013, pp. 1-4.

[7] A. Porch, D. Slocombe, and P. P. Edwardsb, "Microwave absorption in powders of small conducting particles for heating applications," Phys. Chem. Chem. Phys., vol. 15, no. 8, pp. 2757-2763, Feb. 2013.

[8] J. J. Shah, S. G. Sundaresan, J. Geist, D. R. Reyes, J. C. Booth, M. V. Rao, and M. Gaitan, "Microwave dielectric heating of fluids in an integrated microfluidic device," J. Micromech. Microeng., vol. 17, no. 11, pp. 2224-2230, Nov. 2007.

[9] D. Issadore, K. J. Humphry, K. A. Brown, L. Sandberg, D. Weitz, and R. M. Westervelt, "Microwave dielectric heating of drops in microfluidic devices," Lab Chip, vol. 9, no. 12, pp. 1701-1706, Jun. 2009.

[10] K. J. Shaw et al., "Rapid PCR amplification using a microfluidic device with integrated microwave heating and air impingement cooling," Lab Chip, vol. 10, no. 13, pp. 1725-1728, Jul. 2010.

[11] J. J. Shah, G. Jon, and G. Michael, "Microwave-induced adjustable nonlinear temperature gradients in microfluidic devices," J. Micromech. Microeng., vol. 20, no. 10, Oct. 2010, Art. ID 105025.

[12] M. S. Boybay, A. Jiao, T. Glawdel, and C. L. Ren, "Microwave sensing and heating of individual droplets in microfluidic devices," Lab Chip, vol. 13, no. 19, pp. 3840-3846, Oct. 2013.
[13] A. A. Abduljabar, D. J. Rowe, A. Porch, and D. A. Barrow, "Novel microwave microfluidic sensor using a microstrip split-ring resonator," IEEE Trans.Microw. Theory Techn., vol. 62, no. 3, pp. 679-688, Mar. 2014.

[14] A. C. Metaxas and R. J. Meredith, Industrial Microwave Heating. London, U.K.: Peregrinus, 1983.

[15] A. Masood, A. Porch, and D. Barrow, Microwave Resonators for Highly Sensitive Compositional Analysis. Saarbrücken, Germany: Lambert, 2010.

[16] S. Lucyszyn and I. D. Robertson, "Analog reflection topology building blocks for adaptive microwave signal processing applications," IEEE Trans.Microw. Theory Techn., vol. 43, no. 3, pp. 601-611, Mar. 1995.

[17] J. Li, J. Chen, P. Chen, and F. Zhu, "Varactor-tuned half mode substrate integrated waveguide reflection-type phase shifter," in Millim. Wave Wireless Technol. Appl. Microw. Workshop Series, Nanjing, China, 2012, pp. 1-3.

[18] M. Xu, E. Eyring, and S. Petrucci, "Dielectric relaxation of chloroform and chloroform-cyclohexane mixtures at gigahertz and terahertz frequencies: The inertial term," J. Mol. Liq., vol. 73-74, pp. 41-48, Nov. 1997.

[19] S. M. Shameli, T. Glawdel, Z. Liu, and C. L. Ren, "Bilinear temperature gradient focusing in a hybrid PDMS/glass microfluidic chip integrated with planar heaters for generating temperature gradients," Anal. Chem., vol. 84, no. 6, pp. 2968-2973, Mar. 2012.

[20] A. Khayari, M. Medrano, E. Verlage, M. C. Velázquez-Ahumada, M. J. Freire, and A. Ramos, "Microwave-induced water flow in a microchannel built on a coplanar waveguide," J. Appl. Phys., vol. 110, no. 6, Sep. 2011, Art. ID 064912.

[21] R. Somaraju and J. Trumpf, "Frequency, temperature and salinity variation of the permittivity of seawater," IEEE Trans. Antennas Propag., vol. 54, no. 11, pp. 3441-3448, Nov. 2006.

[22] H. Frohlich, Theory of Dielectric. New York, NY, USA: Oxford Univ. Press, 1958.

[23] D. M. Pozar, Microwave Engineering. New York, NY, USA: Wiley, 2005.

[24] G. L. Matthaei, L. Young, and E. M. T. Jones, Microwaoe Filters, Inrpedunce-Matching Networks, and Coupling Structures. New York, NY, USA: McGraw-Hill, 1964.

[25] P. K. Ikalainen and G. L. Matthai, "Wide-band, forward-coupling microstrip hybrids with high directivity," IEEE Trans.Microw. Theory Techn., vol. MTT-35, no. 8, pp. 719-725, Aug. 1987.

[26] S. Park, H. Choi, and Y. Jeong, "Microwave group delay time adjuster using parallel resonator," IEEE Microw. Wireless Compon. Lett., vol. 17, no. 2, pp. 109-111, Feb. 2007.

[27] G. A. Dimitrakis et al., "A system for traceable measurement of the microwave complex permittivity of liquids at high pressures and temperatures," Meas. Sci. Technol., vol. 20, no. 4, pp. 1-6, Feb. 2009.

[28] D. Slocombe, A. Porch, E. Bustarret, and O. A. Williams, "Microwave properties of nanodiamond particles," Appl. Phys. Lett., vol. 102, no. 24, Jun. 2013, Art. ID 244102.

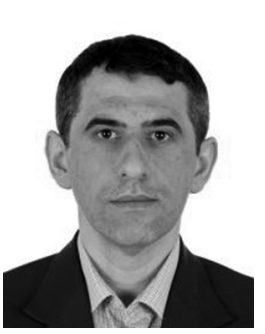

Ali Amin Abduljabar (S'14) received the B.Sc. and M.Sc. degrees in electrical engineering from the University of Basrah, Basrah, Iraq, in 1998 and 2001, respectively, and is currently working toward the Ph.D. degree at Cardiff University, Cardiff, U.K.

$\mathrm{He}$ was a Lecturer on wireless and microwave communications engineering with the University of Basrah. His research concerns the design of microwave sensors for microfluidic systems and noninvasive applications and microwave heating techniques.

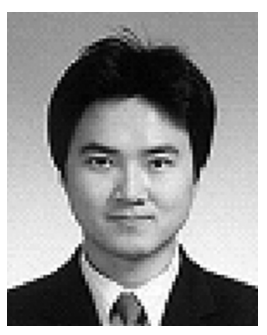

Heungjae Choi (S'06-M'11) received the B.S., M.S., and Ph.D degrees in electronic engineering from Chonbuk National University, Jeonju, Korea, in 2004, 2006, and 2011, respectively.

From 2006 to 2011, he served an alternative military service as a Specialty Research Worker while working toward the Ph.D degree. He is currently a Post-Doctoral Research Associate with the Centre for High Frequency Engineering, Cardiff University, Cardiff, U.K. From 2011 to 2012, he was involved with the development of ultra-fast active harmonic 
load-pull system development as a collaboration with National Instruments and had a successful live demonstration at the 2013 IEEE Radio and Wireless Week, Austin, TX, USA. Since 2013, he has been involved with the development of the noninvasive blood glucose monitoring sensor system and clinical trials funded by the Wellcome Trust. He has authored or coauthored over 50 papers in peer-reviewed journals and conference proceedings. His research interests include microwave circuits, especially high efficiency power amplifiers and linearizers, and their application to material characterization and biomedical engineering.

Dr. Choi has served as a reviewer for IEEE MICROWAVE AND WIRELESS COMPONENTS LETTERS, the IEEE TRANSACTIONS ON CiRCUITS AND SYSTEMS-II: EXPRESS BRIEFS, Progress in Electromagnetics Research, IET Microwaves, Antennas \& Propagation, and the International Journal of Antennas and Propagation. He was the recipient of the Outstanding Achievement Award of the Student High Efficiency Power Amplifier Design Competition, IEEE Microwave Theory and Techniques Society (IEEE MTT-S) International Microwave Symposium (IMS) (2008), and the Samsung Human Tech Thesis Prize Award (2010)

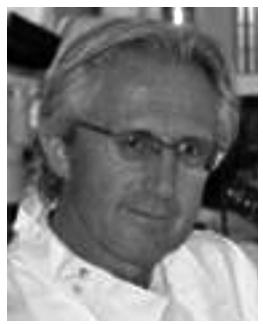

David A. Barrow received the B.Sc. (Hons.) degree in biological sciences and Ph.D. degree in ecological sciences from the University of Wales, Cardiff, U.K.

$\mathrm{He}$ is a currently a multidisciplinary Scientist and Professor of microfluidics with the School of Engineering, Cardiff University. He has researched a diversity microfluidic-based phenomena and devices including chemical sensors, porous silicon, microacoustics, hybrid integration, micromolding, emulsion and digital microfluidics, chemical separations, plasma etching, CFD, microwave sensors, laser micromachining, and marine microanalysis systems. He was a founder of the metaFAB TSB open-access NanoCentre, MSTB Ltd., where he researched space microsystems; the Protasis Corporation, where he developed microdevices for chemical separations; and Q-CHIP Ltd., where he developed injectable microencapsulated pharmaceuticals. His current research interests involve the fabrication of nuclear fusion targets, artificial cells, and stem-cell microcapsules.

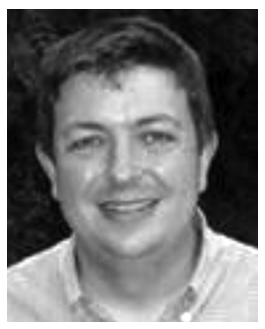

Adrian Porch received the M.A. degree in physics and $\mathrm{Ph} . \mathrm{D}$. degree in low-temperature physics from Cambridge University, Cambridge, U.K.

$\mathrm{He}$ is currently a Professor with the School of Engineering and a member of the Centre for High Frequency Engineering, Cardiff University, Cardiff, U.K. He is also Deputy Director of the School of Engineering, where he is responsible for research and innovation. He possesses over 25 years of experience in applying microwave methods to measure and understand the fundamental properties of electronic materials. More recently, his techniques have been used to develop new types of electromagnetic sensors with an emphasis on applications across different disciplines. 